\title{
Cost-effectiveness analyses
}

\section{Beauty and the beast?}

\author{
Gary R. Cutter, PhD \\ Guogiao Wang, MS
}

I

$\mathrm{n}$ this issue of Neurology ${ }^{\circledR}$ Clinical Practice, Young et al. ${ }^{1}$ discuss 5 things to consider in reading a cost-effectiveness or decision analysis study. Such studies are necessary as the medical profession wrestles with caring for patients and paying for that care. The approaches discussed by Young et al. are important to digest as this type of study will be more prevalent as pressures mount in all aspects of clinical and comprehensive care. Young et al. point out the need to interpret the models and understand the meaning of the data presented. Their article provides an excellent summary of the tools and approaches widely used by policymakers and researchers, bringing some systematic evaluation to the pressures of cost vs the benefits of therapy.

Armed with the materials from the Young et al. article, readers should also be cognizant of limitations that can result from mathematical subtleties hiding within these methods. Knowing the methods and terminology can be as important as reading the literature.

There are many methods used to manage and assess risk and costs. Risk-sharing schemes are methods to limit expenditures relative to the medical gain that can come from a therapy or an intervention. These so-called schemes inform payer's decisions about coverage and denial of therapies and technologies, and usually are built around cost-effectiveness analysis. While they are more widely used in the European Union in a formal process, clinicians everywhere will face these issues when trying to obtain payment for new therapies. Thus, clinicians should understand at some level the basis of the decision-making by the payers. Understanding specifically — or even generally — why a payer has taken a particular action can greatly help to facilitate appeals and discussions.

As an example, consider a risk-sharing scheme that was developed in the United Kingdom for treating multiple sclerosis (MS). An article commenting on this risk-sharing scheme from a few years ago focused on the cost-effectiveness of disease-modifying treatments (DMTs) in relapsing-remitting $\mathrm{MS}^{2}$ and offered the following conclusions:

Editorial, page 372 See page 413

Department of Biostatistics, UAB School of Public Health, Birmingham, AL.

Funding information and disclosures are provided at the end of the article. Full disclosure form information provided by the authors is available with the full text of this article at Neurology.org/cp.

Correspondence to: cutterg@uab.edu 


\section{Risk-sharing schemes are methods to limit expenditures relative to the medical gain that can come from a therapy or an intervention.}

"This 2-year interim analysis of the UK risk-sharing scheme does not provide reliable evidence on cost-effectiveness of disease-modifying treatments.

Results are highly sensitive to how the baseline score and missing follow-up data are handled.

Longer, more complete follow-up with additional reference datasets will be more informative and less sensitive to short-term fluctuations in disability."

These conclusions, while stated in reference to the particular investigation of the costeffectiveness of DMTs, actually apply more broadly. First, they emphasize the limitations of using short-term data for issues that require a long-term perspective as with the question of the long-term effect of treatments on the course of MS. There are short-term and long-term objectives with most treatments. Short-term effects are easier to consider, or measure, or compare, because of the time frame of reference. Long-term outcomes present many more complexities in data collection, compliance, participation, and potentially intervening events that confound therapies. For example, a recurring criticism of the relationship between Betaseron and its effect on mortality at 21 years $^{3}$ is that the investigators did not know what treatments the participants took and for how long they took them over the post trial period. Additionally, as pointed out by Boggild et al., ${ }^{2}$ decisions on how to handle the missing follow-up information make such long-term investigations difficult. This problem is lessened in the Goodin et al. ${ }^{3}$ mortality article, because registries of deaths could be tapped, minimizing missing information. Nevertheless, whenever there are missing data, the results of a study can be mired in what-if controversies.

Short-term results are important for certain questions. Do steroids limit or reduce the effects of relapses? What are the effects of various treatments on postherpetic neuralgia (PHN) pain ${ }^{4,5}$ ? In these situations, there is a risk that long-term safety and long-term effects are sacrificed for the immediate costs or effectiveness results. For such clinical situations, comparing the costeffectiveness on immediate clinically important results answers practical, clinical, and formulary questions.

There are multiple pitfalls readers make when reading the literature. Two key ones are over-reliance on $p$ values and being seduced by percent reductions in event rates. $p$ Values are the probability that the observed result is due to chance, when the null hypothesis is true. $p$ Values tell us how often we are wrong, not how true the results are. While $p$ values are not directly imbedded in cost-effectiveness studies, such studies are rarely conducted on treatments or therapies that do not exhibit statistically significant (i.e, $p$ values) results. Thus, remembering that $p$ values tell us how often we are wrong, it is clear that interpreting them strictly as the strength of the evidence is faulty. $p$ Values are a direct function of the sample size. The larger the sample size, the smaller the $p$ value for the same treatment difference or effect. For example, if we conduct a trial in PHN pain, and the proportion of patients who have a $50 \%$ or more reduction in pain score in the placebo arm of the PHN trial is $14 / 116=12 \%$, and this rate is compared to an active arm, which has $34 / 110=$ $31 \%$ with a $50 \%$ or more reduction in pain, we would have a $p$ value $<0.0006$. These results and $p$ value would occur by chance 6 times in 10,000 if the true success rates were equal. However, if we had only 25 placebo subjects with a success rate of $12 \%(3 / 25)$ and $31 \%$ successes among 35 active subjects, the resulting $p$ value for the same 2 event rates would be $p=0.122$. The estimates of the treatment effects are the same, but the $p$ values are orders of magnitude different. 


\section{p Values are a direct function of the sample size. The larger the sample size, the smaller the value for the same treatment difference or effect.}

Keeping both long-and short-term limitations as well as $p$ value pitfalls in mind, consider the important concern expressed by Boggild et al. ${ }^{2}$ on the sensitivity of the results to the baseline values. This is a critical component of interpreting trial results as well as costeffectiveness studies. It is not discussed at length by Young et al., ${ }^{1}$ but is implicit in many cost-effectiveness studies. We emphasize it here because it is an important concept often lost in marketing endeavors, or by those who wish to emphasize the positive or negative results when the absolute differences are small.

The so-called base rate issue results from the fact that summary reductions are derived from calculations of differences relative to the absolute size of the rates seen in the reference group (often the placebo arm of a trial). Consider 2 pivotal trials in MS, the AFFIRM trial of natalizumab and the FREEDOMS trial of fingolimod. In AFFIRM, the annualized relapse rate in the placebo arm was found to be 0.73 . This is considered the base from where to measure the reduction in relapse rates, hence the term "base rate." The natalizumab arm experienced an annualized relapse rate of 0.23 . The percentage reduction in relapse rate can be derived by computing the difference in relapse rates, called the absolute reduction or absolute risk reduction, and dividing it by the base rate, or $(0.73-0.23) / 0.73=0.50 / 0.73=68.5 \%$. Thus, the absolute reduction in relapse rates was 0.50 with a percent reduction of $68.5 \%$. In FREEDOMS, the placebo arm experienced an annualized relapse rate of 0.40 , its base rate, and the fingolimod arm experienced an annualized relapse rate of 0.18 . This leads to a percentage reduction of $(0.40-0.18) / 0.40=0.22 / 0.40=55 \%$ with an absolute reduction of 0.22 . Thus, the percentage reductions were similar (68.5\% compared to $55 \%)$.

Making a comparison of absolute risk reductions in a manner that offers a more clinically meaningful interpretation can be done by comparing the number needed to treat (NNT), or the number of patients that need to be treated to save one outcome event. The NNT is defined as $1 /$ absolute difference in rates. Thus, the NNT for natalizumab is $1 /(0.50)=2$ compared to the NNT for fingolimod of $1 /(0.22)=4.5$. This means that for every 2 patients treated with natalizumab, we would estimate that one relapse is prevented compared to the NNT with fingolimod of 4.5 patients treated in order to save one relapse.

This example shows that similarity in percentage reductions results from using different base rates. The fact that the absolute risk reductions are more than double for natalizumab compared to fingolimod is obscured unless one looks at the base rates. In addition, it is important to consider the base rates themselves. In this example, in the placebo arms, base rates are markedly different (the rate in the fingolimod trial is about half of that seen in the natalizumab trial). Thus, making simple statements about absolute risk reduction is equally in need of caution as emphasizing the importance of base rates in discussing percent reductions.

The cautionary notes about base rates can be summarized as follows: If one compares the percentage reduction in annualized relapse rates, these 2 drugs show $68.5 \%-55 \%$. Using the absolute difference or the NNT, we see a larger effect for natalizumab either because the number of relapses prevented is greater (absolute difference) or the NNT is smaller, implying that a benefit is achieved with treatment of fewer patients. Finally, if we compare the annualized relapse rates of the treatment arms directly, fingolimod shows a 0.18 annualized relapse rate compared to the 0.23 annualized relapse rate of natalizumab. Thus, fingolimod has a lower annualized relapse rate by almost $22 \%$ compared to natalizumab. 
Comparing absolute rates across studies also requires considering the differences in the studies, and especially the base rates that allow a comparison of the same treatments. Thus, the base rate matters and, as we can see from these 2 studies, comparisons within a study make more sense than comparisons among or between studies.

Thus, the message of cost-effectiveness as well as other analyses is that a single study, a single effect, or a single comparison may simply not be sufficient to establish either the benefit of a therapy or the cost-effectiveness of a therapy, even when effectiveness of a therapy has been demonstrated. The methods of cost-effectiveness are solid, but need careful consideration. They require thoughtful understanding as they represent more than simple calculations.

\section{REFERENCES}

1. Young KC, Kelly AG, Holloway RG. Reading a cost-effectiveness or decision analysis study: five things to consider. Neurol Clin Pract 2013;3:413-420.

2. Boggild M, Palace J, Barton P, et al. Multiple sclerosis risk sharing scheme: two year results of clinical cohort study with historical comparator. BMJ 2009;339:b4677.

3. Goodin DS, Reder AT, Ebers GC, et al. Survival in MS: a randomized cohort study 21 years after the start of the pivotal IFN $\beta-1 b$ trial. Neurology 2012;78:1315-1322.

4. Rowbotham MC, Harden N, Stacey B, Bernstein P, Magnus-Miller L. Gabapentin for treatment of postherpetic neuralgia. JAMA 1998;280:1837-1843.

5. Rice ASC, Maton S; Post Herpetic Neuralgia Study Group. Gabapentin in postherpetic neuralgia; a randomised, double-blind, controlled study. Pain 2001;94:215-224.

\section{STUDY FUNDING}

No targeted funding reported.

\section{DISCLOSURES}

G. Cutter has served on Data and Safety Monitoring Committees for Apotek, Biogen-Idec, Cleveland Clinic, Glaxo Smith Klein Pharmaceuticals, Gilead Pharmaceuticals, Modigenetech/Prolor, Merck/ Ono Pharmaceuticals, Merck, Neuren, PCT Bio, Revalesio, Sanofi-Aventis, Teva, Vivus, NHLBI (Protocol Review Committee), NINDS, NMSS, and NICHD (OPRU oversight committee); has served as a consultant and on advisory boards and received speaker honoraria from Alexion, Allozyne, Bayer, Celgene, Coronado Biosciences, Consortium of MS Centers (grant), Diogenix, Klein-Buendel Incorporated, Medimmune, Novartis, Nuron Biotech, Receptos, Spiniflex Pharmaceuticals, and Teva Pharmaceuticals; receives funding from NIH; and is President of Pythagoras, Inc., a private consulting company located in Birmingham, AL. G. Wang reports no disclosures. Full disclosure form information provided by the authors is available with the full text of this article at Neurology.org/cp.

\section{What Do You Think?}

Discover what your colleagues in clinical practice are talking about. And then add to the discussion!

Neurology ${ }^{\circledR}$ Clinical Practice welcomes your feedback on our articles and seeks your practical, real-world experience on how you face opportunities and challenges in clinical practice. Correspondence regarding articles that appear in Neurology: Clinical Practice may be sent via e-mail to NCPjournal@neurology.org or by clicking on the "Respond" button in the right column of a full text version of an article. 